\title{
Guía de práctica clínica. Prevención de parto pretérmino
}

\author{
José A. Ramírez-Calvo ${ }^{1 *}$, Karla P. Lara-Guerrero², Berenice Velázquez-Torres ${ }^{1}$, Juan M. Gallardo-Gaona ${ }^{1}$, \\ Sandra Acevedo-Gallegos ${ }^{1}$ y Dulce $M^{a}$ Camarena-Cabrera ${ }^{1}$ \\ ${ }^{1}$ Departamento de Medicina Fetal, Instituto Nacional de Perinatología Isidro Espinoza de los Reyes, Ciudad de México; ${ }^{2}$ Departamento de Medicina \\ Materno Fetal, Hospital de la Mujer de Puebla, Puebla. México
}

\section{Resumen}

Se realizó la presente guía de práctica clínica (GPC) como base para el desarrollo de la clínica de parto pretérmino del Departamento de Medicina Fetal del Instituto Nacional de Perinatología y su publicación pretende dar a conocer las bases en la mejor evidencia que fundamentan las acciones de predicción, prevención y tratamiento en todas las pacientes que acuden al departamento.

Se describen las características de la guía:

- Clasificación de la enfermedad: parto prematuro.

- Categoría de GPC: prevención.

- Usuarios potenciales: médicos generales, médicos familiares y médicos ginecoobstetras.

- Población blanco: mujeres embarazadas con riesgo de parto pretérmino asintomáticas.

- Intervenciones y actividades consideradas: medidas generales, uso de progesterona, fibronectina fetal, ultrasonido: longitud cervical, cerclaje cervical, pesario.

- Utilidad clínica: identificar pacientes con alto riesgo para desencadenar parto pretérmino, utilidad de técnicas actuales para predecir parto pretérmino con alta probabilidad.

Palabras clave: Parto pretérmino. Tamizaje. Prevención. Guía de práctica clínica.

\section{Prevention of preterm delivery. Clinical practice guidelines}

\section{Abstract}

This clinical practice guide (CPG) was made as a basis for the development of the preterm delivery clinic of the Department of Fetal Medicine of the National Institute of Perinatology and its publication aims to publicize the foundations in the best evidence that underlies the actions of prediction, prevention and treatment in all patients who are attended at the department. The characteristics of the guide are described:

- Classification of the disease: premature delivery.

- CPG category: prevention.

- Potential users: general practitioners, family doctors and obstetrician gynecologists.

- Target population: pregnant women with risk of preterm delivery asymptomatic.

\section{Correspondencia:}

*José Antonio Ramírez-Calvo

E-mail: tonoramirez@gmail.com

Open Access bajo la licencia CC BY-NC-ND (http://creativecommons.org/licenses/by-nc-nd/4.0/).
Disponible en internet: 25-03-2019 Perinatol Reprod Hum. 2019;33:23-36

www.perinatologia.mx 
- Interventions and activities considered: general measures, use of progesterone, fetal fibronectin, ultrasound: Cervical length measurement, cervical cerclage, pessary.

- Clinical utility: identify patients at high risk to develop preterm birth, utility of current techniques to predict preterm delivery with high probability.

Key words: Preterm birth. Screening. Prevention. Clinical practice guideline.

\section{Clasificación}

- Clasificación de la enfermedad: parto prematuro.

- Categoría de guía de práctica clínica (GPC): prevención.

- Usuarios potenciales: médicos generales, médicos familiares y médicos ginecoobstetras.

- Población blanco: mujeres embarazadas con riesgo de parto pretérmino asintomáticas.

- Intervenciones y actividades consideradas:

- Medidas generales.

- Uso de progesterona.

- Fibronectina fetal.

- Ultrasonido: longitud cervical.

- Cerclaje cervical.

- Pesario.

- Utilidad clínica: identificar pacientes con alto riesgo para desencadenar parto pretérmino, utilidad de técnicas actuales para predecir parto pretérmino con alta probabilidad.

- Estrategia de búsqueda: se realizó una búsqueda en las bases de datos Medline, Embase, Cochrane y PubMed con las palabras clave «clinical practice guidelines», «progesterone», «preterm birth prevention», «cerclaje», «risk factors for preterm birth», "cervical length», «fetal fibronectin» y «pessary». Se incluyeron estudios realizados en humanos, en inglés y español. Se obtuvo un total de 6,748 estudios, de los cuales se utilizaron aquellos con la mejor calidad metodológica.

\section{Preguntas a responder}

- ¿Cuáles son los datos clínicos que identifican pacientes con alto riesgo de desencadenar parto pretérmino?

- ¿Existe entre las técnicas actuales de diagnóstico alguna con alta probabilidad de predicción de parto pretérmino?

- ¿Cuál es la eficacia de la medición de la longitud cervical por ultrasonido para identificar pacientes con riesgo de parto pretérmino?

- ¿Es de utilidad la medición de la longitud cervical por ultrasonido en pacientes asintomáticas de bajo riesgo como predictor de parto pretérmino?
- ¿Es de utilidad el empleo de la fibronectina fetal en pacientes asintomáticas de alto y bajo riesgo como predictor de parto pretérmino?

- ¿Qué medidas son de utilidad como profilaxis en pacientes identificadas con riesgo de parto pretérmino?

\section{Aspectos generales}

\section{Introducción}

El parto pretérmino representa un problema obstétrico y de salud pública. Contribuye hasta en un $70 \%$ a la mortalidad perinatal mundial, con alta morbilidad neonatal tanto inmediata como a largo plazo, manifestada esta última por secuelas (sobre todo neurológicas) que generan un gran costo para las instituciones, la sociedad, los gobiernos y sobre todo para las familias. Por tanto es imperante conocer los métodos diagnósticos actuales que permitan identificar de forma oportuna a las pacientes con riesgo de parto pretérmino".

A pesar de los avances en la atención perinatal, la incidencia de parto prematuro sigue en aumento, sobre todo por la relación con embarazos múltiples asociados a técnicas de reproducción asistida ${ }^{2}$.

La importancia de este documento radica en la atención de un problema muy frecuente, que ocasiona altos costos y saturación de las Unidades de Terapia Intensiva Neonatal, cuyo propósito es ser una herramienta para estandarizar métodos diagnósticos para prevención de parto pretérmino en el segundo y el tercer nivel de atención'.

Existe evidencia clara que demuestra como algunas intervenciones realizadas en el campo de la salud pueden incrementar el tiempo de gestación y así ayudar a madurar al feto y disminuir la morbimortalidad asociada a esta patología y una guía clínica basada en evidencia puede facilitar el paso de la investigación a la práctica ${ }^{2,3}$.

\section{Marco teórico}

La prematurez representa la primera causa de morbimortalidad neonatal en nuestro país y en el mundo; su frecuencia varía entre un 5 y un $12 \%$ de los partos. El riesgo de morir de un recién nacido prematuro es 180 veces mayor que el de un recién nacido a término, y 
aquellos prematuros que sobreviven tienen un mayor riesgo de secuelas e invalidez. Este grupo explica el $83 \%$ de la mortalidad perinatal y los nacidos a las 28 semanas o menos de gestación, el $66 \%$ de las muertes.

La prematurez es causante del $50-60 \%$ de la mortalidad neonatal y del $50 \%$ de las alteraciones neurológicas ${ }^{4}$. Es responsable de complicaciones neonatales a corto plazo tales como síndrome de dificultad respiratoria, hemorragia intraventricular, sepsis, trastornos metabólicos, enterocolitis necrotizante, ductus arterioso persistente, displasia broncopulmonar y apneas. $\mathrm{Y}$ a largo plazo, de complicaciones como parálisis cerebral, retraso mental, compromiso de la visión y pérdida de audición $n^{4,5}$.

Un tratamiento adecuado de las mujeres embarazadas con riesgo de parto prematuro permite prolongar la vida intrauterina, optimizando las condiciones del feto para el nacimiento, sobre todo cuando la prematurez es extrema, lo que se traduce en niños de menos de $1,500 \mathrm{~g}$ y/o menores de 32 semanas al nacer.

Una tercera parte de los partos prematuros son de causa materna (iatrógenos), otro tercio corresponde a pacientes con membranas rotas y el otro tercio a pacientes con membranas íntegras asociadas principalmente a isquemia o infección ${ }^{2,6}$.

\section{Justificación}

El parto pretérmino ocurre en aproximadamente el $8 \%$ del total de nacimientos en EE.UU. y alcanza el $15 \%$ en países subdesarrollados; mundialmente se presenta en aproximadamente el $10 \%$ de los partos. La Organización Mundial de la Salud (OMS) reporta alrededor de 13 millones de nacimientos pretérmino por año, el $84 \%$ de estos nacimientos ocurren entre las 32-36.6 semanas; en África y Asia ocurre el $85 \%$ de estos nacimientos. En México la tasa de parto pretérmino es del $8.6 \%$, siendo el $35 \%$ de estos los que ocurren de forma espontánea sin factores de riesgo asociados ${ }^{1}$.

Es la principal causa de morbimortalidad, se ha asociado a riesgo de parálisis cerebral, ceguera, retraso mental, sordera y retinopatía, comparado con recién nacidos a término.

El $75 \%$ de las muertes perinatales son atribuibles a prematurez, un recién nacido (RN) que pesa menos de $1,500 \mathrm{~g}$ tiene un riesgo de morir dentro de su primer año de vida de 180 veces superior a la de un $\mathrm{RN}$ con peso mayor a 2,500 g. A nivel mundial el riesgo de muerte de un prematuro es 20 veces superior al de un $\mathrm{RN}$ a término.

La incidencia de parto pretérmino es generalmente más alta en los grupos socioeconómicos bajos, por ello la prevención continúa siendo un objetivo en la obstetricia, quedando claro que este parto pretérmino es resultado de una gran variedad de factores, como sustancias o agentes infecciosos, embarazos múltiples y antecedentes de parto prematuro previo. Los partos prematuros son más frecuentes en mujeres de 35 años o mayores, en situaciones de pobreza, con otras patologías agregadas. Cerca del $50 \%$ de los partos pretérmino son potencialmente prevenibles, además de presentar factores modificables como tabaquismo, estrés, vaginosis bacteriana o infección de tracto urinario, bacteriuria asintomática y empleo de drogas ilícitas. El desafío consiste en integrar estrategias e intervenciones eficaces en un proceso de atención continua materna y neonatal en los establecimientos de sa$\operatorname{lud}^{4,7}$. A pesar de que existen instrumentos para asignar riesgo de presentar complicaciones basados en antecedentes clínicos, sus resultados resultan subóptimos, ya que solo seleccionan a un pequeño grupo de pacientes del total de las cuales una intervención oportuna podría prevenir el padecimiento. Así, implementar programas de tamizaje a todas las embarazadas independientemente del riesgo asignado con anterioridad basado en la medición de la longitud cervical puede mejorar las intervenciones dirigidas, como el empleo de progesterona, pesario o cerclaje, para disminuir la tasa de parto pretérmino, reducir el número de ingresos hospitalarios innecesarios y el consumo de recursos institucionales, disminuir la angustia de la paciente y su familia y, en caso de ya contar con datos clínicos de parto pretérmino, poder realizar acciones oportunas para intentar mejorar los resultados perinatales.

Finalmente, todo el personal de salud debe participar de estas acciones independientemente del nivel de atención en el que se encuentre, con referencia oportuna de la paciente sin riesgo aparente de parto pretérmino a lugares donde existan programas de predicción y prevención ${ }^{4,8}$.

\section{Objetivos}

1. Establecer si los factores de riesgo clínicos son de utilidad para predecir parto pretérmino.

2. Determinar si existe entre las técnicas de diagnóstico actuales alguna con alta probabilidad de predicción de parto pretérmino.

3. Valorar la eficacia de la medición de la longitud cervical por ultrasonido como método diagnóstico para identificar pacientes con alta probabilidad de parto pretérmino.

4. Valorar la eficacia de la medición de la longitud cervical por ultrasonido en pacientes asintomáticas de bajo riesgo como predictor de parto pretérmino. 
5. Determinar la eficacia de la fibronectina fetal en pacientes asintomáticas de alto y bajo riesgo como predictor de parto pretérmino.

6. Identificar medidas profilácticas o preventivas en pacientes con alto riesgo de parto pretérmino.

\section{Evidencias y recomendaciones}

Las recomendaciones aquí señaladas son producto del análisis de las guías de práctica clínica internacionales seleccionadas mediante el modelo de revisión sistemática de la literatura. Corresponden a la información disponible organizada según criterios relacionados con las características cuantitativas, cualitativas, de diseño y tipo de resultados de los estudios que las originaron.

Los niveles de las evidencias y la graduación de las recomendaciones se mantienen respetando la fuente original consultada. Las evidencias se clasifican de forma numérica y las recomendaciones con letras; ambas, en orden decreciente de acuerdo a su fortaleza $a^{9,10}$.

\section{GRADOS DE EVIDENCIA Y RECOMENDACIÓN: CENTRE Evidence-Based Medicine de OXford (CEBM)}

- Evidencia

- Ia: Evidencia obtenida de revisiones sistemáticas y metaanálisis de investigaciones controladas y aleatorizadas.

- lb: Evidencia obtenida de al menos una investigación controlada y aleatorizada, con intervalo de confianza estrecho.

- Ila: Evidencia obtenida por lo menos de un estudio bien diseñado sin aleatorización, revisión sistemática de estudio de cohorte, con homogeneidad.

- Ilb: Evidencia obtenida por lo menos de un estudio bien diseñado casi experimental, cohortes.

- III: Evidencia obtenida de estudios descriptivos no experimentales, estudios comparativos, estudios de correlación de casos y controles.

- IV: Evidencia obtenida de informes u opiniones de expertos y/o experiencia clínica de autoridades respetadas.

- Grado de recomendación

- A: Extremadamente recomendable.

- B: Recomendación favorable.

- C: Recomendación favorable pero no concluyente.

- D: Sin evidencia adecuada de investigación. Ni se recomienda ni se desaprueba ${ }^{9,10}$.

\section{Definiciones}

La OMS define el parto pretérmino como el nacimiento antes de las 37 semanas de gestación completas (259 días), después del primer día de la fecha de última regla.

- Temprano: < 32 semanas de gestación.

- Tardío: > 32 semanas de gestación.

- Prematuro extremo: < 28 semanas de gestación.

\section{Epidemiología}

El parto pretérmino es un problema de salud pública mundial. Se calcula que nacen en el mundo 13,000,000 de niños antes de término, con cifras de prematurez que varían en los distintos continentes: el continente europeo, con un $6.2 \%$, y el continente americano (norte), con un $10.6 \%$, son los que lo presentan con menor frecuencia. En nuestro país las cifras se mantienen entre el 8 y el $10 \%$. El parto pretérmino es una enfermedad multifactorial y estos factores pueden darse de forma independiente o conjuntarse y desencadenar parto pretérmino no solo en el actual embarazo, sino también en los sucesivos.

Se describen tres entidades clínicas bien definidas relacionadas con el parto prematuro:

- Amenaza de parto pretérmino con membranas íntegras.

- Rotura prematura de membranas.

- Complicaciones propias del embarazo/iatrogénicas.

Esta guía se refiere a la primera de ellas, siendo las restantes motivo de otras publicaciones.

\section{Prevención}

Los profesionistas sanitarios se enfrentan cada vez más a un trabajo de equipo multidisciplinario, con competencias en continuo desarrollo, por lo que sus decisiones y prácticas deben estar basadas en la mejor evidencia disponible, identificando las intervenciones más efectivas y accesibles en términos de costo que puedan tener un impacto sobre la calidad de vida ${ }^{2,10}$.

Las acciones que desarrollar desde los tres niveles de atención son las siguientes:

- Prevención primaria. Se refiere a todas aquellas acciones que se realicen a fin de eliminar o disminuir factores de riesgo en la población general. Podemos actuar en etapa preconcepcional o en etapas tempranas del embarazo.

- Prevención secundaria. Acciones de diagnóstico y prevención tempranos de enfermedades recurrentes en personas con riesgo demostrado, es decir, aquellas pacientes que ya presentaron un 
embarazo con un parto pretérmino o que presentan otros factores de riesgo.

- Prevención terciaria. Son las acciones destinadas a reducir la morbilidad y la mortalidad después de que se ha producido el daño, es decir, una vez que se ha desencadenado una amenaza de parto pretérmino o trabajo de parto pretérmino ${ }^{4}$.

\section{Prevención primaria}

\begin{tabular}{|c|c|c|c|}
\hline & Estrategia diagnóstica & $\begin{array}{l}\text { Grado de } \\
\text { recomendación }\end{array}$ & Nivel de evidencia \\
\hline \multirow{6}{*}{ Preconcepción } & $\begin{array}{l}\text { Se recomienda una consulta preconcepcional a toda la } \\
\text { población de mujeres en edad fértil en la que se realizarán } \\
\text { acciones de promoción y prevención de la salud destinadas } \\
\text { a detectar condiciones de salud general que puedan ser } \\
\text { optimizadas, como corregir hábitos alimentarios y controlar/ } \\
\text { prevenir sobrepeso y obesidad, que aumenta el riesgo de } \\
\text { parto prematuro }\end{array}$ & $\begin{array}{l}\text { D } \\
\text { Guía clínica prevención } \\
\text { parto prematuro. } \\
\text { MINSAL }^{8}, 2010 \\
\operatorname{lams}^{5}, 2008\end{array}$ & $\begin{array}{l}\text { Ila } \\
\text { Manuck }{ }^{11}, 2011\end{array}$ \\
\hline & $\begin{array}{l}\text { De igual manera se controlará la adicción al alcohol, } \\
\text { drogas o tabaco }\end{array}$ & $\begin{array}{l}\text { C } \\
\text { Guía clínica prevención } \\
\text { parto prematuro. } \\
\text { MINSAL }^{8}, 2010 \\
\text { Bukowski }^{12}, 2009\end{array}$ & $\begin{array}{l}\text { Ib } \\
\text { Vidaeff, } 2006 \\
\text { Gray }^{12}, 2001\end{array}$ \\
\hline & $\begin{array}{l}\text { La fortificación preconcepcional con ácido fólico durante } \\
\text { un año antes del embarazo disminuye la posibilidad de } \\
\text { parto prematuro en un } 5 \text { a } 7 \%\end{array}$ & & $\begin{array}{l}\text { la } \\
\text { Boutin }{ }^{13}, 2012\end{array}$ \\
\hline & $\begin{array}{l}\text { Prevenir el embarazo en adolescentes y los no planeados, } \\
\text { promover planeación e intervalo entre cada gestación. } \\
\text { Prevenir, descartar y tratar enfermedades de transmisión } \\
\text { sexual tiene RR: } 0.77 \text { (IC 95\%: 0.56-1.05) para parto prematuro, } \\
\text { pero su tratamiento no lo disminuye. Realizar tamizaje para } \\
\text { diagnosticar y manejar padecimientos mentales, tamizaje } \\
\text { para diagnóstico y manejo de enfermedades crónicas }\end{array}$ & $\begin{array}{l}\text { C } \\
\text { OMS, } 2012 \\
\text { B } \\
\text { SOGC. Clinical Practice } \\
\text { Guideline, } \\
2011 \\
\text { ACOG }^{15}, 2012\end{array}$ & \\
\hline & $\begin{array}{l}\text { La enfermedad periodontal tiene un RR para parto } \\
\text { prematuro de } 0.89 \text { (IC } 95 \%: 0.73-1.08 \text { ), sin embargo el } \\
\text { tratamiento no disminuye el parto prematuro }\end{array}$ & & \\
\hline & $\begin{array}{l}\text { Las pacientes con antecedente de parto pretérmino deben } \\
\text { recibir asesoramiento preconcepcional y atención médica } \\
\text { temprana en el embarazo }\end{array}$ & & \\
\hline Concepción & Estrategia diagnóstica & Grado de recomendación & Nivel de evidencia \\
\hline \multirow{4}{*}{ Factores de riesgo } & $\begin{array}{l}\text { Establecer edad gestacional por la fecha de última } \\
\text { menstruación (FUM) presenta errores. Por tanto se debe } \\
\text { calcular mediante la medición de la longitud craneocaudal } \\
\text { por ultrasonido en el primer trimestre } \\
\text { En todas las mujeres embarazadas se realizará búsqueda } \\
\text { de factores de riesgo durante el control prenatal }\end{array}$ & $\begin{array}{l}\text { B } \\
\text { Geirsson }{ }^{16}, 1997\end{array}$ & $\begin{array}{l}\text { III } \\
\text { OMS, } 2012 \\
\text { la } \\
\text { México: Secretaría } \\
\text { de Salud }^{1}, 2008 \\
\text { Spong }^{17}, 2007\end{array}$ \\
\hline & $\begin{array}{l}\text { El factor de riesgo más importante para predicción de } \\
\text { parto pretérmino es el antecedente de parto pretérmino } \\
\text { previo OR: } 2.0\end{array}$ & $\begin{array}{l}\text { B } \\
\text { SOGC. Clinical Practice } \\
\text { Guideline, } \\
2011\end{array}$ & $\begin{array}{l}\text { I-III } \\
\text { ACOG }^{15}, 2012\end{array}$ \\
\hline & $\begin{array}{l}\text { Antecedente de parto pretérmino antes de las } 37 \text { semanas } \\
\text { y longitud cervical corta se considera riesgo del } 16 \% \text { para } \\
\text { un parto antes de las } 37 \text { semanas }\end{array}$ & & \\
\hline & $\begin{array}{l}\text { Riesgo para parto prematuro antes de las } 34 \text { semanas de } \\
\text { gestación: antecedente de } 2 \text { nacimientos entre la semana } \\
16 \text { y } 30,2 \text { nacimientos entre } 16 \text { y } 30 \text { semanas más un } \\
\text { nacimiento de término, antecedente de un parto prematuro }\end{array}$ & & $\begin{array}{l}\text { III } \\
\text { México: IMSS, } 2013 \\
\text { Beta }^{18}, 2011\end{array}$ \\
\hline
\end{tabular}


Riesgo para parto prematuro antes de las 37 semanas de gestación: longitud cervical corta $(25 \mathrm{~mm})$ antes de las 24 semanas, uso de alcohol y otras toxicomanías, antecedente de 2 partos prematuros antes de las 37 semanas, antecedente de 2 partos prematuros entre 32 y 36 semanas

Múltiples ensayos clínicos no han podido demostrar reducción en los partos pretérmino siguiendo estrategias de prevención como: monitoreo en el hogar de la actividad uterina, exámenes digitales cervicales seriados, reposo en cama y educación acerca de los signos y síntomas de trabajo de parto pretérmino entre las mujeres con factores de riesgo identificables

Realizar tamizaje de las características maternas e historia obstétrica entre las 11 y 13.6 semanas puede identificar una tasa de falsos positivos en el 10 a cerca del $20 \%$ de las nulíparas que presentarán parto prematuro
III

México: IMSS, 2013

Robinson ${ }^{19}, 2012$

Argentina: Guía de práctica clínica ${ }^{4}, 2011$

la

Cochrane Database: Sosa ${ }^{20}, 2004$

III México: IMSS, 2013 Beta $^{18}, 2011$

\section{Concepción Prevención} primaria

\section{Estrategia diagnóstica}

Las revisiones sistemáticas han encontrado que ni los suplementos de proteína isocalóricas ni los suplementos equilibrados de proteína/energía son útiles para reducir el riesgo de parto pretérmino, aunque tienen otros beneficios. Suplementos nutricionales: se ha observado que en poblaciones con una alimentación rica en ácidos omega-3 Nutrición

Estilo de vida el embarazo se prolonga una semana en relación a poblaciones con dietas menos ricas en estos ácidos grasos

Una ganancia de peso menor durante el tercer trimestre también podría ser un factor de riesgo para parto prematuro. Un análisis retrospectivo de 7,259 partos encontró que durante los últimos meses del embarazo se asoció con parto prematuro

Estilo de vida y conductas: evitar adicciones, tabaco, alcohol y drogas. Las intervenciones tempranas para dejar de fumar en primer nivel de contacto pueden incrementar de forma significativa el número de mujeres que dejan de fumar o reducen el mínimo de cigarrillos a más del $50 \%$, reduciendo así el número de partos pretérmino

Apoyo social, educación a la mujer embarazada. La evidencia presenta resultados no concluyentes en términos de reducción o prevención de incidencia de parto pretérmino

Reposo en cama, reducción de actividad física, reducción de viajes, reposo en cama. No se ha encontrado evidencia del impacto en la reducción del riesgo al brindar estas recomendaciones. No se ha encontrado evidencia que demuestre que el reposo en cama produzca algún beneficio, así como no existe incremento del riesgo por continuar con actividad física. No se ha encontrado ningún estudio controlado que analice los efectos de la recomendación de guardar reposo en casa frente a reposo intrahospitalario. Existe muy poca información para evaluar los beneficios y daños de recomendar el reposo en cama, la decisión debe ser tomada de manera individual y discutida con la paciente

\section{Grado de recomendación}

(n)

\section{C}

Argentina: Guía de práctica clínica ${ }^{4}, 2011$ Olsen ${ }^{23}, 2004$

IIla

México: Secretaría de Salud', 2008 ICSI, 2008

A México: Secretaría de Salud', 2008 ICSI, 2008

C Sciscione ${ }^{26}, 2010$ la C Sciscione ${ }^{26}, 2010$
Ilb

Khoury ${ }^{21}, 2005$

Catov'2, 2007

\section{Nivel de evidencia}$$
\text { Catover }
$$ 
Estilo de vida

Infección tracto urinario

Vaginosis

bacteriana
El internamiento antenatal para guardar reposo no ha mostrado ser efectivo en disminuir el parto pretérmino y la morbilidad perinatal. Puede presentar efectos adversos como trombosis venosa y atrofia muscular, así como incrementar el estrés en las mujeres, y debe evitarse en la medida de lo posible

Actividad sexual: reducir o eliminar la actividad sexual durante el embarazo argumentando que el orgasmo femenino y el semen produzcan prostaglandinas que puedan precipitar contracciones. Mientras las membranas se encuentren íntegras, no se ha encontrado evidencia que justifique el recomendar la abstinencia sexual para prevenir el parto pretérmino

En la primera consulta de control prenatal, solicitar urocultivo, idealmente a las 12 semanas de gestación. Un metaanálisis que incluyó 12 trabajos con 2011 embarazadas demostró disminución significativa del parto pretérmino (RR: 0.50; IC 95\%: 0.365-0.698) en el grupo de portadoras de bacteriuria asintomática que recibe tratamiento con antibiótico

El cultivo de orina obtenido de las 12 a las 16 semanas de gestación puede identificar un $80 \%$ de mujeres que tendrán bacteriuria asintomática en el embarazo con un 1-2\% adicional cuando se realiza el tamizaje mensual

El tamizaje y tratamiento sistemático con antibióticos de la bacteriuria asintomática durante el embarazo es recomendable

Evidencia de estudios controlados y aleatorizados indica un incremento en el riesgo entre bacteriuria asintomática e incremento de riesgos fetales, como parto prematuro, entre mujeres no tratadas y mujeres sin bacteriuria (RR: 1.25$)$

Existe evidencia del incremento en el riesgo de parto pretérmino en mujeres con bacteriuria asintomática que no han recibido tratamiento comparado con mujeres que no tienen bacteriuria asintomática. La diferencia del riesgo oscila entre el 2.1 y el $12.8 \%$

Una revisión sistemática de 14 estudios controlados y aleatorizados comparó el tratamiento con antibiótico frente a ningún tratamiento o placebo. El tratamiento con antibiótico redujo la bacteriuria persistente durante el embarazo (OR: 0.070; IC 95\%: 0.05-0.10), con menor riesgo de parto pretérmino (OR: 0.60; IC 95\%: 0.45-0.80)

Existe evidencia que sugiere que la vaginosis bacteriana se asocia con un riesgo de parto prematuro 2 a 6 veces mayor

La vaginosis bacteriana se asocia con parto pretérmino. En una revisión de casos y controles y estudios de cohorte se encontró que las mujeres con vaginosis bacteriana tenían 1.8 veces más probabilidades de tener partos pretérmino que las mujeres sin infección

En mujeres asintomáticas y mujeres sin factores de riesgo identificados para parto pretérmino no se recomienda tamizaje de rutina para tratar la vaginosis bacteriana Mujeres con alto riesgo de parto pretérmino pueden beneficiarse al realizar el tamizaje de rutina y el tratar de vaginosis bacteriana
B Sciscione ${ }^{26}, 2010$

C

Costa Rica: Guías de práctica clínica ${ }^{7}, 2006$

A Argentina: Guía de IIa práctica clínica ${ }^{4}, 2011$

Guía clínica.

MINSAL $^{8}, 2010$

Smaill26, 2007

C

México: Secretaría de Salud', 2008.

Golberg, 2008

A Cochrane Database Smaill ${ }^{26}, 2004$ México: Secretaría de Salud', 2008 Tita, 2007

$\mathrm{lb}$ México: Secretaría de Salud ${ }^{1}, 2008$ McPheeters ${ }^{27}$, 2008

la México: Secretaría de Salud, 2008

II

$\mathrm{ACOG}^{28}, 2008$

$\mathrm{ACOG}^{28}, 2008$

B

$\mathrm{ACOG}^{28}, 2008$

III

México: Secretaría de Salud ${ }^{1}, 2008$. Mc Donald ${ }^{29}, 2007$
B

México: Secretaría de Salud', 2008

Mc Donald ${ }^{29}, 2007$ 
Perinatol Reprod Hum. 2019;33

\section{Prevención secundaria}

\section{Concepción \\ Prevención \\ secundaria \\ Estrategia diagnóstica}

Longitud cervical por USG Bajo riesgo
El riesgo de parto pretérmino es inversamente proporcional a la longitud cervical medida por ultrasonido

Se recomienda utilizar un valor de corte de $15 \mathrm{~mm}$ para población de bajo riesgo a la hora de iniciar medidas de prevención

Cuando el cuello es menor de $15 \mathrm{~mm}$ (presente en el $1 \%$ de la población general) identifica el $30 \%$ de la población que tendrá parto antes de las 35 semanas; se incrementa el riesgo de parto pretérmino entre 6 y 9 veces

La ecografía transabdominal no debe utilizarse para la evaluación de la longitud cervical para predecir el parto prematuro en pacientes de bajo riesgo

La longitud cervical se puede usar para identificar embarazadas asintomáticas de menos de 24 semanas de gestación. Ante la posibilidad de algunos medios que han logrado predecir parto prematuro y ante el alto costo que este implica, se puede considerar el tamizaje universal de la medición de la longitud cervical vía vaginal si se cuenta con el recurso material y personal capacitado

La ecografía TV tiene un alto valor predictivo negativo si la longitud cervical es de $3 \mathrm{~cm}$ o más después de las 24 semanas de gestación. Esta información debe ser utilizada para evitar intervenciones innecesarias

La medición de cérvix a las 22-24 semanas en población general permite identificar a la población de mayor riesgo de parto prematuro

La longitud cervical en la población obstétrica en general se mantiene estable dentro de los 2 primeros trimestres. La historia natural de los cambios de la longitud cervical puede ser utilizada para identificar pacientes con riesgo incrementado para parto prematuro

El ultrasonido transvaginal debe utilizarse para evaluar pacientes con antecedente de parto prematuro para distinguirlas entre alto y bajo riesgo de presentar nuevo parto prematuro y puede ser útil en la prevención de una intervención innecesaria

Longitud cervical por USG

Alto riesgo
La longitud cervical entre las 11 y 13.6 semanas es más corta en pacientes con antecedente de parto prematuro

La longitud cervical presenta cambios mínimos entre las 11 y 24 semanas; en caso de presentarse son más evidentes en pacientes con parto prematuro

La longitud cervical debe realizarse entre las semanas 20.1 a 34 en pacientes con riesgo de parto prematuro

Se recomienda utilizar un valor de corte de $25 \mathrm{~mm}$ para población de alto riesgo

\section{Grado de recomendación Nivel de evidencia \\ Nivel de ovidencia}

(2)

D

SOGC, 2011

Ramírez/Lara, 2013

II-2

México: Secretaría de Salud' 2009 SOGC, 2011

II-2

Kagan $^{30}, 2006$

SOGC, 2011

IIa

IIa

ACOG $^{15}, 2012$.

SOGC, 2011

Ramírez/Lara,

2013.

A

$\operatorname{Lim}^{31}, 2011$

B

Kagan $^{29}, 2006$

Ila

SOGC, 2011

Guidelines for the management of spontaneous preterm labor, 2011

II a México: Secretaría de Salud, 2009 SOGC ${ }^{1}, 2011$

A

II-2

SOGC, 2011

III

México: IMSS' 12013

Beta $^{18}, 2011$

III

México: IMSS ${ }^{1}, 2013$

Souka, 2011
México: Secretaría de

Salud, 2009

Iglesias, 2007

A

$\operatorname{Lim}^{31}, 2011$ 
Una longitud cervical $<25 \mathrm{~mm}$ se relaciona con el incremento del riesgo de parto prematuro, con una $\mathrm{OR}$ de 0.97. (IC 95\%: 0.26-1.32). Entre las mujeres con un cuello uterino corto, por cada milímetro de acortamiento cervical entre ultrasonidos aumentan un $3 \%$ las probabilidades de tener parto prematuro

No existe consenso sobre el tiempo óptimo o la frecuencia de las evaluaciones de la longitud cervical, si las mediciones se repiten deben hacerse a intervalos apropiados para minimizar la probabilidad de error de observación

La longitud cervical puede ser utilizada para identificar riesgo incrementado de parto pretérmino en mujeres asintomáticas con $<24$ semanas de gestación que tienen otros factores de riesgo para parto pretérmino. Sin embargo, hay evidencia insuficiente para recomendar estrategias de manejo específicas como el cerclaje para estas pacientes

No hay ensayos aleatorizados específicos que hayan evaluado intervenciones en mujeres asintomáticas con embarazo de $>24$ semanas que tengan riesgo incrementado de parto y que tienen una disminución en la longitud cervical. Esta información puede ser de utilidad para el manejo empírico de estas pacientes, incluida la disminución del nivel de actividad, trabajo y viajes, aumento de la vigilancia y la administración de corticoides

Realizar medición de longitud cervical entre las 20-24 semanas en pacientes con riesgo de parto pretérmino, si se encuentra bajo el percentil 5 ( $\geq 25 \mathrm{~mm}$ ); se repetirá a los 14 días. Si disminuye su longitud más de $8 \mathrm{~mm}$ se recomienda realizar cerclaje.

No existe un consenso sobre la frecuencia o el momento para realizar la ecografía de longitud cervical posterior a la colocación del cerclaje

En mujeres con antecedente de uno o más partos prematuros idiopáticos previos, debe evaluarse la longitud cervical cada 2 semanas entre las $14-24$ semanas. Si se detecta acortamiento progresivo se debe valorar necesidad de cerclaje o uso de progesterona

Longitud cervical por USG

Alto riesgo y bajo riesgo
El ultrasonido transabdominal no debe ser utilizado para evaluar la longitud cervical como predictor de parto prematuro

El ultrasonido endovaginal es el ideal para valorar la longitud cervical

La longitud cervical debe realizarse entre las semanas 20.1 a 34

La ecografía transvaginal puede ser utilizada para evaluar el riesgo de parto prematuro en mujeres con antecedente de parto prematuro espontáneo y diferenciar aquellas de alto riesgo con las de bajo riesgo para parto pretérmino. La edad gestacional de un parto pretérmino previo afecta a la longitud cervical en un embarazo futuro

Existe una correlación precisa entre la longitud cervical medida por ultrasonido vaginal y el riesgo de parto prematuro, y se han establecido valores de corte para determinar qué paciente posee alto o bajo riesgo de parto prematuro
A

$\operatorname{Lim}^{31}, 2011$

A

Ila

$\operatorname{Lim}^{31}, 2011$

SOGC, 2011

Ila

SOGC, 2011

C

SOGC, 2011

SOGC, 2011

B

Iams, 2004

B

Guidelines for the

Ila management of spontaneous preterm labor, 2011

B

SOGC, 2011

A

SOGC, 2011

B

SOGC, 2011
II-2D

SOGC, 2011

SOGC, 2011

\author{
Ila \\ SOGC, 2011
}

A

$\operatorname{Lim}^{31}, 2011$ 
Progesterona Alto y bajo riesgo

Progesterona Alto y bajo riesgo
Se recomienda utilizar un valor de corte de $25 \mathrm{~mm}$ para población de alto riesgo y de $15 \mathrm{~mm}$ para población de bajo riesgo, a la hora de iniciar medidas de prevención

El ultrasonido transperineal para valorar la longitud cervical solo se reserva para aquellos casos en los que no es posible utilizar la vía vaginal

Se requieren mayores estudios en los cuales se evalúen resultados perinatales, así como el impacto en el costo-beneficio de realizar la medición del cérvix de forma sistemática

Con base en la mejor evidencia disponible, una longitud cervical de $20 \mathrm{~mm}$ en pacientes sin antecedente de parto pretérmino previo y de $25 \mathrm{~mm}$ en pacientes con dicho antecedente se asocia con mayor riesgo de parto espontáneo de menos de 35 semanas de gestación

En mujeres con embarazo único sin historia de parto prematuro, con longitud cervical $<20 \mathrm{~mm}$ antes de las 24 semanas, el uso de progesterona vaginal $200 \mathrm{mg}$ se asocia a disminución en el riesgo de parto prematuro

En el examen de las 20-24 semanas, si el cérvix se encuentra bajo el percentil 5, especialmente si el cuello es menor de $15 \mathrm{~mm}$, se debe administrar progesterona natural micronizada

Se recomienda administración de progesterona natural micronizada $200 \mathrm{mg} /$ día por vía vaginal, desde el diagnóstico de cuello corto hasta las 36 semanas

Si existen antecedente de uno o más partos prematuros menores de 35 semanas, se beneficiarán con la administración de progesterona

Las mujeres sin antecedentes de parto prematuro y con cérvix menor de $15 \mathrm{~mm}$ se benefician con la administración de progesterona vaginal

La hidroxiprogesterona por vía intramuscular reduce la frecuencia de parto prematuro en pacientes asintomáticas que presentan un cérvix de $15 \mathrm{~mm} 0$ menos

La longitud cervical aunada a progesterona $200 \mathrm{mg} / 24 \mathrm{~h}$ disminuye el riesgo de parto prematuro antes de las 34 semanas en un $34.4 \%$, y en pacientes con antecedente de parto prematuro y longitud cervical menor a $28 \mathrm{~mm}$ disminuye riesgo de parto prematuro antes de las 32 semanas en un $29.6 \%$

En pacientes con embarazo único y antecedente de parto prematuro se recomienda el uso de $250 \mathrm{mg}$ de caproato de 17 alfa hidroxiprogesterona intramuscular de forma semanal iniciando desde la semana 16 a las 26 en caso de identificar una longitud cervical menor de $25 \mathrm{~mm}$ antes de las 24 semanas

El uso de progesterona de forma suplementaria protege contra el parto prematuro recurrente

El empleo de progesterona en mujeres con antecedente de parto prematuro disminuye de forma significativa la probabilidad de parto, antes de las 34 semanas con RR de 0.58 y antes de las 37 semanas de 0.8

En todas las pacientes, el antecedente de parto prematuro y/o longitud cervical menor de $15 \mathrm{~mm}$ entre las 22 y 26 semanas de gestación es indicación para iniciar terapia a base de progesterona
II-2D

SOGC, 2011

IA

Cochrane Database.

Berguella ${ }^{33}, 2013$

III

Berguella ${ }^{33}, 2013$

A

México: IMSS' 2013

Berguella ${ }^{34}, 2012$

México: IMSS' 2013

Berguella ${ }^{34}, 2012$

A

SOGC, 2011

A

Sánchez-Ramos ${ }^{35}, 2009$

A

Da Fonseca, 2003

Argentina: Guía de práctica clínica ${ }^{4}, 2011$

A Argentina: Guía de práctica clínica ${ }^{4}, 2011$

A Argentina: Guía de práctica clínica ${ }^{4}, 2011$

B

México: IMSS', 2013

Berguella ${ }^{34}, 2012$

IB

SOGC, 2011

III

México: IMSS, 2013.

Robinson ${ }^{19}, 2018$

la

México: IMSS1', 2013

Dodd, 2012

A

SOGC, 2011

SOGC, 2011 
Fibronectina fetal Bajo riesgo

Fibronectina fetal Alto riesgo

Cerclaje
Existe evidencia que demuestra que una única muestra cervicovaginal en el segundo trimestre con un resultado positivo para niveles de fibronectina tiene un valor moderado para predecir parto prematuro, pero un resultado negativo disminuye la probabilidad de parto pretérmino espontáneo solo mínimamente

La combinación de FNF con ultrasonido es recomendable de forma secuencial y puede ser más eficaz que una sola de las pruebas. Sin embargo, se requiere mayor investigación al respecto

No se sugiere realizar la prueba de fibronectina fetal en mujeres asintomáticas

Las pacientes sintomáticas con alto riesgo de parto prematuro con un resultado negativo es poco probable que experimenten nacimiento pretérmino en los siguientes siete días. El beneficio de la prueba de fibronectina fetal es que tiene un alto VPN $91 \%$

Deberá realizarse entre las semanas 24 a 34 de gestación en pacientes con antecedente parto pretérmino

El test para fibronectina posee una especificidad del $84 \%$ y una sensibilidad del $56 \%$ para un punto de corte de $50 \mathrm{ng} / \mathrm{ml}$

Si la longitud cervical es de 21-30 mm, deberá medirse la fibronectina fetal en secreción vaginal, ya que la combinación de ambos cuenta con una sensibilidad del $86 \%$ y una especificidad del $90 \%$, con un VPP del $63 \%$ y un VPN del $97 \%$, contando con una capacidad de predicción de parto prematuro para los siguientes 28 días

La aplicación de cerclaje en la población asintomática sin antecedentes de parto prematuro y cuello acortado entre 20-24 semanas no reduce la incidencia de parto prematuro

En mujeres asintomáticas con antecedente de parto pretérmino una longitud cervical menor de $25 \mathrm{~mm}$ antes de las 24 semanas de gestación aunado a cerclaje cervical disminuye de forma significativa la incidencia de parto pretérmino del 39 al 23\%, con RR: 0.61 (IC 95\%: 0.40-0.92)

Cuando el cerclaje se compara con no dar tratamiento en caso de longitud cervical menor $25 \mathrm{~mm}$, el RR para parto pretérmino es 0.80 (IC 95\%: 0.69 a 0.95)

El cerclaje estaría indicado solo en ausencia de infección

En pacientes con antecedente de parto prematuro espontáneo y cuello acortado $<15 \mathrm{~mm}$ antes de las 23 semanas, la aplicación de cerclaje disminuye el riesgo de parto pretérmino con RR de 0.23 (IC 95\%: 0.08-0.66)

En las mujeres asintomáticas con una historia de parto pretérmino previo espontáneo y ecografía con $\mathrm{LC}<25 \mathrm{~mm}$ antes de la semana 24, el cerclaje cervical debe ser considerado para reducir el riesgo de parto pretérmino

No está claro si la ecografía para LC tiene ventajas significativas sobre el examen clínico posterior a la colocación de un cerclaje, no existe un consenso sobre la frecuencia o el momento para realizar el ultrasonido después del cerclaje
$\mathrm{Ib}$

México: Secretaría de Salud', 2008.

Berguella ${ }^{34}, 2008$

Ila

SOGC, 2011

$\mathrm{Ib}$

México: Secretaría de Salud', 2008. Berguella $^{34}, 2008$

A

Berguella $^{34}, 2008$

Ib

Berguella ${ }^{34}, 2008$

Conde-Agudelo ${ }^{37}$, 2011

Audibert $^{38}, 2010$

la

SOGC, 2011

III

México: IMSS'1 2013

B

Vidaeff, 2009

$\mathrm{Ib}$

SOGC, 2011

C

Drakeley ${ }^{24}, 2008$

Ib

Alfirevic ${ }^{39}, 2012$

IV

Guidelines for the management of spontaneous preterm labor, 2011

B

Argentina: Guía de práctica clínica ${ }^{4}, 2011$ Vidaeff, 2009

B

SOGC, 2011

la

SOGC, 2011

Ilb

SOGC, 2011 
Perinatol Reprod Hum. 2019;33

Pesario

Solo un estudio prospectivo y aleatorizado ha confirmado utilidad en cuanto a prevención, con una disminución del $28 \%$, por lo que esta estrategia debe ser utilizada aún con cautela

El uso de pesario reduce la incidencia de parto prematuro antes de las 37 semanas del 22 al $59 \%$ si se compara solo con manejo expectante (IC $95 \%$ : $0.27-0.49$ ), y antes de las 34 semanas del $27 \%$ (IC $95 \%$ : $0.13-0.43$ ) cuando se coloca de las 18 a las 22 semanas
Dharan $^{40}, 2009$

Ib

Alemm, 2013

Goya, 2012

\section{Conflicto de intereses}

Los autores declaran que no existe conflicto de intereses.

\section{Responsabilidades éticas}

Protección de personas y animales. Los autores declaran que para esta investigación no se han realizado experimentos en seres humanos ni en animales.

Confidencialidad de los datos. Los autores declaran que en este artículo no aparecen datos de pacientes.

Derecho a la privacidad y consentimiento informado. Los autores declaran que en este artículo no aparecen datos de pacientes.

\section{Bibliografía}

1. Guías de práctica clínica. Diagnóstico y manejo del parto pretérmino. México: Secretaría de Salud; 2009.

2. Guía Clínica. Prevención de parto prematuro. Santiago de Chile: Ministerio de Salud (MINSAL); 2005.

3. Beck S, Wojdyla D, Say L, Betran AP, Merialdi M, Harris Requejo J, et al The worldwide incidence of preterm birth: a systematic review of maternal mortality and morbidity. Bull World Health Organ. 2010;88:31-8.

4. Guía de Práctica clínica. Amenaza de Parto Pretérmino. Argentina. 2011.

5. lams JD, Romero R, Culhane JF, Goldenberg RL. Primary, secondary and tertiary interventions to reduce the morbidity and mortality of preterm birth. Lancet. 2008;371(9607):164-75.

6. Lim K, Butt K, Crane, JM. No. 257-Ultrasonographic cervical length assessment in predicting preterm birth in singleton pregnancies. J Obstet Gynaecol Can. 2018;40(2):e151-64.

7. Criterios Técnicos y Recomendaciones Basadas en evidencia para la Construcción de Guías de Práctica Clínica. Tratamiento y Prevención de Parto Pretérmino. Costa Rica. 2006.

8. Guía Clínica. Prevención de parto prematuro. Santiago de Chile: Ministerio de Salud (MINSAL); 2010.

9. Guyatt GH, Oxman AD, Vist GE, Kunz R, Falck-Ytter Y, Alonso-Coello P, Schünemann HJ; GRADE Working Group. GRADE: an emerging consensus on rating quality of evidence and strength of recommendations. BMJ. 2008 Apr 26;336(7650):924-6

10. Higgins JPT, Green S (editors). Cochrane Handbook for Systematic Reviews of Interventions Version 5.1.0 [updated March 2011]. The Cochrane Collaboration, 2011.

11. Manuck TA, Henry E, Gibson J, Varner MW, Porter TF, Jackson GM, et al. Pregnancy outcome in a recurrent preterm birth prevention clinic. Am J Obstet Gynecol. 2011;204(4):320.

12. Bukowski R, Malone FD, Porter FT, Nyberg DA, Comstock $\mathrm{CH}$ Hankins GD, et al. Preconceptional folate supplementation and the risk of spontaneous preterm birth: A cohort study. PLoS Med. 2009; 6(5):e1000061.

13. Gray R, Wabwire F. Randomized trial of presumptive sexually transmitted disease therapy during pregnancy. Am J Obstret Gynecol. 2001; 185(5):1209-17.
14. Boutin A, Demers S, Roberge S, Roy-Morency A, Chandad F, Bujold E. Treatment of periodontal disease and prevention of preterm birth: systematic review and meta-analysis. Am J Perinatol. 2013;30(7):537-44

15. American College of Obstetricians and Gynecologists. ACOG Practice Bulletin No.130: Prediction and prevention of preterm birth. Obstet Gynecol. 2012;120(4):964-73.

16. Geirsson RT. Ultrasound: the rational way to determine gestational age. Fetal Med Rev. 1997;9:133-46.

17. Spong CY. Prediction and prevention of recurrent spontaneus preterm birth. Obstet Gynecol. 2007;110(2 Pt 1):405-15.

18. Beta J, Akolekar R, Ventura W. Prediction of spontaneous preterm delivery from maternal factors obstetric history and placental perfusion and function at 11-13 weeks. Prenat Diagn. 2011;31:75-83.

19. Robinson JN, Norwitz ER. Preterm birth: Risk factors, interventions for risk reduction, and maternal prognosis. Up to Date. Dec 2018.

20. Sosa C, Althabe F, Belizan J, Bergel E. Bed rest in singleton pregnancies for preventing preterm birth. Cochrane Database Syst Rev. 2004: CD003581.

21. Khoury J, Henriksen T, Seljeflot I, Mørkrid L, Frøslie KF, Tonstad S. Effects of an antiatherogenic diet during pregnancy on markers of maternal and fetal endothelial activation and inflammation: the CARRDIP study. BJOG. 2007:114:279-88.

22. Catov JM, Bodnar LM, Ness RB, Markovic N, Roberts JM. Association of periconceptional multivitamin use and risk of preterm or small-for-gestational-age births. Am J Epidemiol. 2007;166:296.

23. Olsen SF. Is supplementation with marine omega-3 fatty acids during pregnancy a useful tool in the prevention of preterm birth? Clin Obstet Gynecol. 2004;47(4):768-74.

24. Drakeley AJ, Roberts D, Alfirevic Z. Cervical stitch (cerclage) for preventing pregnancy loss in women. Cochrane Database Syst Rev. 2003;1: CD003253.

25. Sciscione A. Maternal activity restriction and the prevention of preterm birth. Am J Obstet Gynecol. 2010;202:232.

26. Smaill FM, Vazquez JC. Antibiotics for asymptomatic bacteriuria in pregnancy. Cochrane Database Syst Rev. 2015 Aug 7;(8):CD000490.

27. Mc Pheeters M, Miller W, Hartmann K. The epidemiology of threatened preterm labor: a prospective cohort study. Am J Obstret Gynecol. 2005; 192:325.

28. Mc Donald H, Brocklehurst $P$, Gordon A. Antibiotics for treating bacteterial vaginosis in pregnancy. Cochrane Database Syst Rev. 2007;(1): CD000262.

29. Society for Maternal Fetal Medicine Publications Committee. ACOG Committee Opinion number 419 October 2008 (replaces no. 291, November 2003). Use of progesterone to reduce preterm birth. Obstet Gynecol. 2008;112(4):963-5.

30. Kagan K, To M, Tsoi E, Nicolaides K. Preterm birth: the value of sonographic measurement of cervical length. BJOG. 2006;113(Suppl. 3):52-6.

31. Lim A, Hegeman A, Huis M, Opmeer BC, Bruinse HW, Mol BW. Cervical length measurement for the prediction of preterm birth in multiple pregnancies: a systematic review and bivariate meta-analysis. Ultrasound Obstet Gynecol. 2011;38(1):10-7.

32. lams JD, Cebrik D, Lynch C, Behrendt N, Das A. The rate of cervical change and the phenotype of spontaneous preterm birth. Am J Obstet Gynecol. 2011;205:130.

33. Berghella V, Figueroa D, Szychowski JM, Owen J, Hankins GD, lams JD, et al.; Vaginal Ultrasound Trial Consortium. 17-alpha-hydroxyprogesterone caproate for the prevention of preterm birth in women with prior preterm birth and a short cervical length. Am J Obstet Gynecol. 2010; 202(4):351.e1-6

34. Berghella V, Hayes E, Visintine J, Baxter JK. Fetal fibronectin testing for reducing the risk of preterm birth. Cochrane Database Syst Rev. 2008;(4):CD006843.

35. Sanchez-Ramos L, Delke I, Zamora J, Kaunitz AM. Fetal fibronectin as a short-term predictor of preterm birth in symptomatic patients: a meta-analysis. Obstet Gynecol. 2009;114:631. 
36. Da Fonseca EB, Bittar RE, Carvalho MH, Zugaib M. Prophylactic administration of progesterone by vaginal suppository to reduce the incidence of spontaneous preterm birth in women at increased risk: a randomized placebo-controlled double-blind study. Am J Obstet Gynecol. 2003; 188:419-24.

37. Conde-Agudelo A, Papageorghiou AT, Kennedy SH, Villar J. Nove biomarkers for the prediction of the spontaneous preterm birth phenotype: a systematic review and meta-analysis. BJOG. 2011;118(9): 1042-54.
38. Audibert F, Fortin S, Delvin E, Diemli A, Brunet S, Dubé J, et al. Contingent use of fetal fibronectin testing and cervical length measurement in women with preterm labour. J Obstet Gynaecol Can. 2010; 32:307-12.

39. Alfirevic Z, Stampalija T, Roberts D, Jorgensen AL. Cervical stitch (cerclage) for preventing preterm birth in singleton pregnancy. Cochrane Database Syst Rev. 2012;(4): CD008991.

40. Dharan VB, Ludmir J. Alternative treatment for a short cervix: the cervical pessary. Semin Perinatol. 2009;33:338-42. 


\section{Anexo 1}

\section{Técnica para medición de la longitud cervical}

1. Vejiga vacía.

2. Explicación a la paciente sobre el procedimiento.

3. Colocar a la paciente en posición de litotomía dorsal posterior.

4. Introducir transductor vaginal, cubierto con preservativo y dirigirlo a fórnix anterior. Se debe evitar ejercer presión sobre el cérvix porque podría aumentar la longitud artificialmente.

5. Obtener un corte sagital del cérvix y utilizar la mucosa endocervical como guía para identificar el orificio cervical interno real, evitando confusiones con el segmento inferior del útero.

6. Magnificar la imagen por lo menos $2 / 3$ de la pantalla, para visualizar el orificio interno y externo adecuadamente.

7. Se utilizan los calipers para medir la distancia lineal entre la zona triangular ecodensa del orificio cervical externo y la zona en forma de « $\mathrm{V} »$ del orificio cervical interno.

8. Para medir la longitud cervical se traza una línea entre los calipers colocados a nivel de los orificios cervicales interno y externo.

9. El primero es donde se junta el canal cervical y el saco amniótico o el polo de la presentación fetal.

10. El orificio cervical externo es el punto donde se unen los labios anterior y posterior del cérvix.

11. Realizar por lo menos tres mediciones, la variación entre las distintas mediciones debe ser menor de $4 \mathrm{~mm}$. Debe anotarse la medida más corta.

12. Cada ecografía debe durar 2-3 min. En aproximadamente el $1 \%$ de los casos la longitud cervical puede cambiar debido a contracciones uterinas. 